HISTORIA Y CONSERVACIÓN

DEL PATRIMONIO EDIFICADO

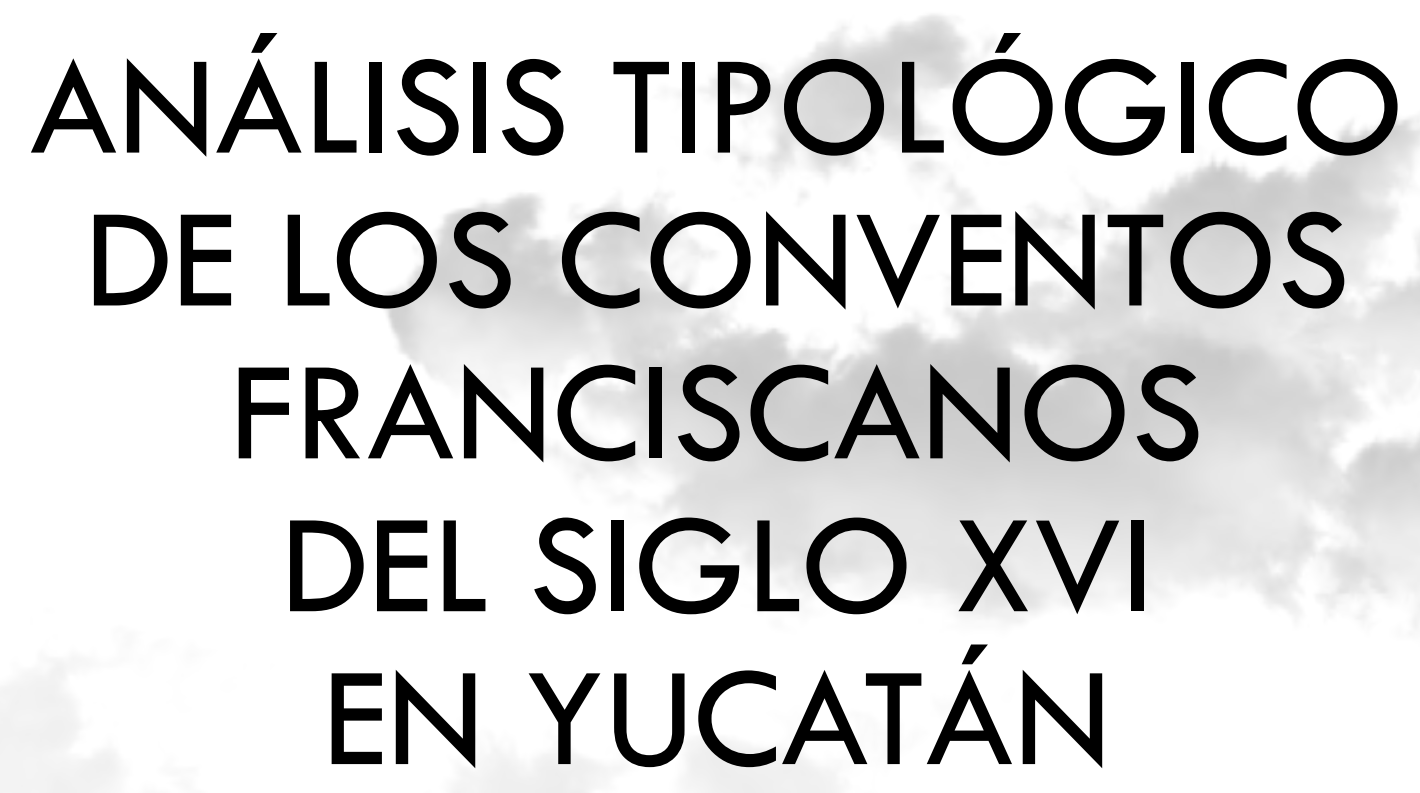




\title{
ANÁLISIS TIPOLÓGICO DE LOS CONVENTOS FRANCISCANOS DEL SIGLO XVI EN YUCATÁN
}

\author{
TYPOLOGICAL ANALYSIS OF THE 16TH CENTURY FRANCISCAN \\ CONVENTS IN YUCATAN
}

Arq. Mario Antonio León Flores

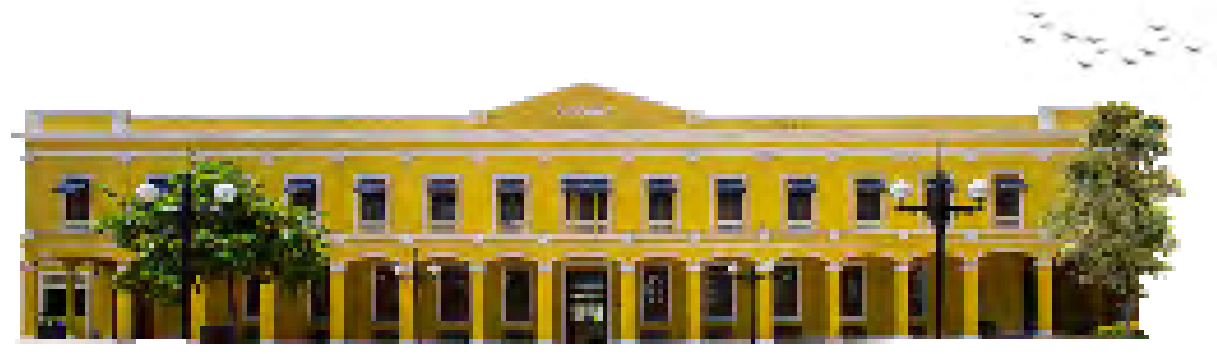

Fecha de envío:

Fecha de aceptación: 9 de mayo de 2019

\section{Resumen:}

Este trabajo dirige su atención a un sector del gran patrimonio cultural con que se cuenta en el país, al de los conventos franciscanos fundados durante el siglo XVI en la península de Yucatán. Estudia en ellos la configuración que tienen las organizaciones formales de sus conjuntos arquitectónicos. El análisis tipológico formal de estos conjuntos conventuales se convierte en un útil instrumento para profundizar en el estudio de sus características formales y para ordenar las relaciones que se presentaron entre las condiciones en que se desarrollaron los procesos de materialización de las obras y las formas concretas que estas adoptaron.

\section{Objetivo General}

Realizar una propuesta metodológica de observación y sistematización para el análisis formal de los conjuntos conventuales que nos ayude a conocer las características formales esenciales de sus partidos arquitectónicos.

\section{Particulares}

Identificar el patrón de configuración de los conjuntos conventuales construidos al inicio de la Colonia en la Península de Yucatán. Identificar las características formales esenciales de los partidos arquitectónicos que los conjuntos conventuales pudieron adoptar en sus procesos de creación.

\section{Summary:}

This work directs its attention to a sector of the great cultural heritage that exists in the country, to that of the Franciscan convents founded during the 16th century in the Yucatan Peninsula. Studies the configuration that formal organizations have of their architectural ensembles. The formal typological analysis of these conventual complexes becomes a useful instrument to deepen the study of their formal characteristics and to order the relationships that arose between the conditions in which the processes of materialization of the works were developed and the concrete forms that they adopted.

\section{- General Objective}

Carry out a methodological proposal for observation and systematization for the formal analysis of the conventual complexes that help us to know the essential formal characteristics of their architectural parts.

\section{口 Individuals}

Identify the configuration pattern of the conventual complexes built at the beginning of the Colony in the Yucatan Peninsula. Identify the essential formal characteristics of the architectural parties that the conventual complexes could adopt in their creative processes.

\section{Palabras Clave:}

Conventos, Franciscanos, Arquitectura, Colonia, Yucatán.

\section{Key words:}

Convents, Franciscans, Architecture, Colonial, Yucatan. 


\section{6}

La investigación histórica arquitectónica regional requiere de sus propios enfoques, sus propias herramientas teóricas y sus propias metodologías.

La manera de aproximarnos al estudio formal del objeto arquitectónico específico que se propone en este trabajo, nos lleva al plano de la proyectación arquitectónica, parte intelectual del proceso creativo pues propone estudiar las organizaciones formales de los conjuntos conventuales y los principios de orden que siguen para vincular e integrar en unidades totalizadoras sus elementos componentes. Este análisis formal de los edificios proporciona elementos para el análisis compositivo a los talleres de proyectos. También pretende proporcionar elementos metodológicos y al mismo tiempo didácticos para ejemplificar conceptos de diseño arquitectónico en las aulas.

Al hacer el estudio tipológico de los conventos, primeramente analizamos dos series tipológicas, la funcional y la formal. La primera, en relación a las funciones que cada instalación desempeñó, como parte del sistema de conventos, en las diferentes etapas del período estudiado. La serie tipológica formal se desarrolla a partir de las organizaciones formales de los conjuntos arquitectónicos. Y establecemos las relaciones entre ambas. Mayor amplitud y complejidad representa el análisis tipológico formal, en él, al analizar las características formales de los diferentes casos de estudio para determinar las características de los tipos y subtipos, también se determinan las variantes que se presentan en algunos casos. Al final se analizan las relaciones que pudieron existir entre las manifestaciones formales de los objetos estudiados (características típicas y variantes) con las condiciones, particulares y generales, que se presentaron en el desarrollo de los procesos.

Esperamos aportar más elementos para la historiografía arquitectónica regional. $Y$ aunque se concentra en aspectos propios de la composición arquitectónica puede servir para vincular las maneras adoptadas en la solución formal de los edificios con los factores condicionantes de tipo social que envolvieron y a la vez vivieron y generaron los principales promotores de la arquitectura religiosa de Yucatán. 


\section{REFERENCIAS TEÓRICAS}

Para eludir el peligro de tomar el estudio de la historia como "fuente de inseguridad", del que nos advierte Tafuri, ${ }^{1}$ es necesario saber lo que pretendemos obtener de ella, lo que nos otorga su comprensión. No abandonarla y pretender que no existe, o que no nos afecta en el proceso de diseño y comprensión de la arquitectura del pasado y de la contemporánea. Al mismo tiempo es vital tener claro que "la utilización de la noción de la historia carente de juicio es prácticamente nula; nunca es fácil de encontrar y es antieconómico intentarlo".2

Cuando Pablo Chico hace referencia a estos temas lo hace para dejarnos claro que sin la historia no es posible entender la arquitectura y que el estudio de la historia sin un referente teórico no nos permitirá llegar a la apropiación de la esencia de los objetos arquitectónicos. ${ }^{3}$

Al pretender hacer un estudio analítico de los conventos construidos en la segunda mitad del siglo XVI en la península de Yucatán debemos abordar el nivel descriptivo como base para el análisis de los objetos de estudio tratando de identificar las relaciones que se establecen entre el sistema arquitectónico y el sistema mayor al que pertenece y cuyos factores lo condicionan. $Y$ esto lo podemos lograr de una forma más ordenada y efectiva si contamos con un método de análisis arquitectónico para hacerlo.

Marina Waisman, Pablo Chico y otros teóricos especialistas y preocupados en el tema han revisado los diversos métodos que existen, y señalan al análisis tipológico como una de las formas particulares que adquiere el análisis arquitectónico, apuntando la utilidad especial que presenta para el trabajo historiográfico.

Entendemos el estudio del tipo, como una manera de ver la arquitectura, de entenderla y de explicarla a través de la búsqueda de sus rasgos persistentes y característicos. Es una manera de estudiar la arquitectura que acepta que dichos rasgos han

1 Tafuri, Teorias e historia de la arquitectura, p. 89, en Chico, Transformaciones y evolución de... p 120.

2 Gregotti, Juicio histórico y encuadre en una perspectiva contemporánea de la arquitectura del pasado, en Chico, op. cit., p. 120.

3 Chico, op. cit, p. 119. 
sobrevivido en el tiempo hasta convertirse en parte esencial de su esquema pero que a pesar de esa persistencia no son estáticos sino que en aparente continuidad van también sufriendo transformaciones en respuesta coherente a las necesidades y las circunstancias del momento en que se presentan.

En opinión de Pablo Chico, un estudio tendría carácter de tipología arquitectónica si incluye análisis de series, muestras, y secuencias suficientes para llegar a conclusiones en relación con las variantes y los elementos funcionales, espaciales, estructurales, expresivos y ambientales. ${ }^{4}$

Es incuestionable la complejidad del fenómeno arquitectónico y que su estudio completo requiere considerarlo en toda esa complejidad, sin embargo proponemos una aproximación al estudio de los edificios que se enfoque inicialmente al análisis tipológico formal general y de conjunto, que visualice los vínculos que existen con los otros aspectos de la arquitectura y deje abiertos los caminos para complementarse con posteriores investigaciones.

\section{ASPECTOS METODOLÓGICOS}

\section{Las series tipológicas}

De acuerdo al planteamiento que hace Marina Waisman, en el estudio de aquellos aspectos específicos del producto arquitectónico, tales como la forma, la función, la estructura, la relación con el entorno, etc., resulta conveniente ordenarlos en series tipológicas que "se desarrollan en el tiempo y en el espacio". ${ }^{5}$

Las series que propone son:

- Series de tipologías formales

- Series de tipologías funcionales

- Series de tipologías estructurales

- Series de tipologías de relación obra/entorno

- Series de tipologías de modos de empleo de las técnicas ambientales

Para efectos de un análisis particular nos concretamos a estudiar dos de ellas, las funcionales y las formales, sin dejar, por ello, de considerar la presencia e importancia que las otras tienen.

4 Ibidem., p. 685

5 Waisman, La estructura histórica..., p. 61. 


\section{Análisis de las series funcionales y formales}

- El análisis funcional se hace a partir de la definición del subgénero arquitectónico y de sus componentes así como de las particulares funciones que le correspondió a cada edificio en la estrategia de evangelización.

- El análisis formal se hace siguiendo el estudio de sus procesos en la categoría territorial. El tipo se analiza formalmente comparando las manifestaciones que tienen los objetos arquitectónicos del mismo sub-género arquitectónico en distintos lugares del territorio en un período específico de su desarrollo.

Este mismo sistema estructural del análisis tipológicoarquitectónico propone que es necesario revisar las relaciones que se dan entre las series mismas y entre estas y aspectos de la realidad socio-cultural en la que se inscriben. Por lo que simultáneamente estaremos haciendo un análisis que pretenda encontrar relaciones entre las expresiones tipológicas y los condicionamientos generales y particulares de dichos procesos.

\section{La matriz elemental}

Al ser nuestro estudio más inclinado a los aspectos de la forma, y por lo tanto de las series formales, nos resulta de vital importancia la reconstrucción de las matrices elementales y observar su desarrollo hasta el tipo, ubicado este en el período definido.

Caniggia define la matriz elemental como el esquema original que subyace en las edificaciones, es el esquema del proyecto básico que podemos encontrar a través del examen del proceso tipológico. ${ }^{6}$

En el análisis tipológico formal vamos a comenzar por encontrar esas matrices elementales, que serían los esquemas que integren las formas arquitectónicas esenciales del convento yucateco del siglo XVI. Este punto es importante de explicar y acotar porque puede parecer relativo. Relativo con respecto a lo que consideramos esencial en la forma del objeto que estamos queriendo analizar.

Si el objeto de estudio es el conjunto de edificios conventuales que los franciscanos construyeron en la segunda mitad del siglo $\mathrm{XVI}$ en Yucatán, nos parece que es imprescindible considerar, en su proceso de desarrollo arquitectónico, la disposición y

6 Caniggia, Tipología de la edificación..., p.33 
organización de sus componentes funcionales más importantes, aquellos elementos arquitectónicos espaciales imposibles de olvidar para la misión que querían y debían cumplir.

En la búsqueda de las formas arquitectónicas esenciales debemos recordar que cuando se inicia el proceso evangelizador formalmente en Yucatán se está al filo de la mitad del siglo XVI, ya han transcurrido aproximadamente 25 años desde que los primeros franciscanos comenzaron sus trabajos en el altiplano mexicano. Tiempo en el que han acumulado experiencias y recursos para el diseño y construcción de sus instalaciones. Los grandes inventos de la arquitectura del siglo XVI, el atrio y la capilla abierta, ya están en pleno desarrollo y construyéndose en otras provincias de la Nueva España. Los esquemas de organización espacial de las instalaciones conventuales que los religiosos conocían desde sus experiencias en Europa ya están siendo probados y adecuados en estas tierras americanas. Las experiencias constructivas y la percepción del espacio que tienen los indígenas están siendo asimiladas en los procesos creativos de las nuevas ciudades y sus edificios. Aún quedan muchas cosas nuevas por conocer y aprender, y mucho más aún por experimentar y desarrollar en el plano de lo urbano y lo arquitectónico, pero se esta avanzando y la tarea se presenta monumental para un número muy limitado de frailes y pioneros. En esta situación los instrumentos conocidos y probados, los recursos para el diseño y la construcción que han demostrado su eficacia y factibilidad se convierten en herramientas importantes a conservar en el proceso que cada día habría que seguir. En cada nueva obra se integran lo conocido con lo innovador condicionados por una circunstancia de disponibilidad de recursos materiales, muy austera en la península de Yucatán.

Dadas estas condiciones para los procesos de proyección y construcción, consideramos que para los religiosos era importante además de ocupar, dentro de los asentamientos indigenas, un sitio seguro y donde quedara clara su presencia y dominio sobre lo pagano, conservar en el plano de lo arquitectónico algunas características formales que cumplieran requerimientos funcionales y simbólicos mínimos que les permitieran desarrollar adecuadamente sus funciones.

\section{Partido y esquema}

Siguiendo el planteamiento metodológico de Caniggia hemos tratado de reconocer en lo construido, a través de la revisión completa de los casos de estudio y de la investigación historiográfica que nos 
muestra el desarrollo de sus procesos, ${ }^{7}$ las características formales arquitectónicas que podemos considerar esenciales. Estas serían:

- La presencia de algunos espacios indispensables donde desarrollar sus fundamentales tareas.

- La disposición y organización de dichos espacios en el conjunto unitario.

Estas dos características formales constituyen, para algunos teóricos del diseño arquitectónico, elementos suficientes para la construcción del "partí" o "partido", síntesis formal mínima del proyecto arquitectónico. En este punto del método el proceso se vuelve un tanto complejo, pues para llegar a plantear principios generales de la forma es necesario conocer las particularidades del universo de estudio y estas hay que verlas desde la perspectiva del conjunto en general.

Para poder llegar a la construcción de una matriz elemental debemos antes a encontrar los partidos de cada uno los casos de estudio. Y esto sólo es posible hacerlo estableciendo algunas variables observables comunes.

En este trabajo el partido es el medio fundamental para el estudio tipológico formal. Se llega a él después de una revisión y análisis de todos y cada uno de los casos. De la observación minuciosa del proyecto arquitectónico, se abstrae su partido y se elabora el esquema que lo representa.

Esta es la parte inicial y fundamental del proceso pero es necesario, para el análisis que nos proponemos, ir un poco más allá, desarrollar y enriquecer los esquemas precisando las características esenciales antes mencionadas, de tal manera que nos ayuden a clarificar mejor la percepción y facilitar el estudio. En este intento proponemos que en la elaboración de los esquemas de los conjuntos conventuales se señalen sus componentes espaciofuncionales y en ellos se consideren las siguientes características formales básicas:

- Figura o perfil básico

- Dimensión y proporción relativa

- Posición respecto al conjunto y los otros componentes

Entenderemos por esquema arquitectónico la representación gráfica, en conjunto, de las características físicas seleccionadas propias de la forma de los objetos de estudio. Es la expresión

7 Ibidem, p. 31 
gráfica del partido. Su representación será tanto planimétrica como volumétrica. $Y$ en dos diferentes escalas, una de conjunto y otra de cada uno de los componentes.

Los componentes que tomamos en consideración son aquellos que se plantean en la desarticulación del sistema arquitectónico y que también hemos denominado como zonas primarias. Y que a continuación relacionamos:
a. Atrio
b. Templo
c. Capilla de indios
d. Claustro o casa
e. Huerto

\section{ESTUDIO DE LAS SERIES TIPOLÓGICAS}

Es particularmente importante el estudio de las series formal y funcional ya que la categoría de análisis a seguir es de carácter formal-funcional respecto a los partidos arquitectónicos. Funcional porque el criterio de identificación de las zonas primarias parte de la relativa homogeneidad en la actividad general que en ella se desarrolla, y formal ya que las variables observables en los partidos arquitectónicos son relativas al carácter formal que presentan.

\section{Las series funcionales}

En este nivel los objetos de estudio son cada uno de los conjuntos conventuales y el análisis funcional se hace una vez que definimos el subgénero arquitectónico que se estudia, o sea después de revisar que todos los casos coinciden con la definición de conjunto conventual que hemos establecido.

Para la caracterización funcional de cada objeto de estudio tomamos en consideración el papel que le correspondió en el proyecto de evangelización del territorio yucateco o, como califica Robert Ricard ${ }^{8}$ a este proceso, en la "conquista espiritual" de Yucatán.

8 Ricard, La conquista espiritual de México, pass. 
De acuerdo a la distribución de las órdenes y los conventos en el territorio de la Nueva España, Ricard propone tres tipos de misión:

- De ocupación: se refiere a aquellos conventos que "forman una red bastante estrecha, a distancia racional unos de otros, y agrupados en torno a un centro". Seguían y consolidaban la conquista militar.

- De penetración: fueron "aquellas fundaciones precarias de casas esporádicas, en zonas de difícil relieve, de clima penoso, aún no del todo pacificadas o rodeadas de territorios totalmente indómitos". Precedian o acompañaban a la conquista militar.

- Casas de enlace: fueron "las series de conventos que, en vez de presentarse e forma concéntrica alrededor de una casa principal forman una línea, más o menos directa, que liga un grupo cualquiera con la cabecera". ${ }^{9}$

Como se puede apreciar estas diferentes "misiones" cumplieron un papel estratégico en los procesos de formación, consolidación y expansión de los territorios evangelizados, rol que con el desarrollo y transformación del mismo proceso y sus circunstancias pudo ir cambiando. Así, una región que en un inicio estuviera destinada a servir como misión de penetración, con el paso del tiempo y una vez pacificado su territorio podía asumir la función de enlace con nuevas instalaciones que avanzaran aún más hacia regiones más lejanas y anteriormente fuera del control militar.

En la península de Yucatán, durante el proceso de "conquista espiritual" del territorio y su población, las estrategias de inserción y crecimiento de los franciscanos requirieron necesariamente del apoyo de una red de instalaciones religiosas (misiones), cuyas funciones generales en este plan, no se diferencian mucho de las de otras partes de la Nueva España. Dada esta relación nos es posible utilizar la denominación que emplea Ricard, inicialmente para indicar las funciones que se asignaron a las distintas instalaciones conventuales, y luego para proponer una serie tipológica funcional.

\section{Relaciones entre el tiempo y la función (períodos de fundación)}

Durante la segunda mitad del siglo XVI y en la primera década del XVII, se construyeron 28 conventos de diferentes características formales.

9 Ibidem., p. 157 
En los aproximadamente 60 años de este período, que podríamos considerar el inicio del proceso de evangelización del territorio, los asentamientos de religiosos en las diferentes localidades, tanto de blancos pero predominantemente de indigenas, ocurrieron en diferentes circunstancias y tiempos. La principal fuente de información que tenemos para establecer las fechas en que sucedieron estos establecimientos son las "tablas de Capítulo" de la Orden Franciscana, documentos que consultó López de Cogolludo y de las que hace relación en su obra "Historia de Yucatán".

A partir de los datos cronológicos, así como de las diferentes condiciones que se dieron en la sociedad de esa época, el período de estudio se ha dividido en cuatro etapas o períodos cuyos límites están señalados por hechos significativos para la organización franciscana y para el proceso de fundación de conventos.

\begin{tabular}{|c|c|c|}
\hline Período & Fecha & Evento sinificativo \\
\hline $\begin{array}{c}\text { Inicial } \\
1546-1561\end{array}$ & 1546 & $\begin{array}{c}\text { Se inicia la misión } \\
\text { a Yucatán } \\
\text { Primera instalación } \\
\text { formal en Campeche } \\
\text { Se forma la Provincia } \\
\text { de Yucatán y Guatemala }\end{array}$ \\
\hline $\begin{array}{c}\text { Consolidación } \\
1562-1572\end{array}$ & 1562 & $\begin{array}{c}\text { Auto de fe de Maní } \\
\text { Llega el obispo Toral } \\
\text { a Yucatán }\end{array}$ \\
\hline $\begin{array}{c}\text { Muere Toral en México } \\
\text { III } \\
1573-1579\end{array}$ & 1571 & $\begin{array}{c}\text { Landa regresa de } \\
\text { España como obispo } \\
\text { de Yucatán } \\
\text { Muerte de Landa }\end{array}$ \\
\hline $\begin{array}{c}\text { IV } \\
\text { Secularización } \\
1580-1603\end{array}$ & 1602 & $\begin{array}{c}\text { Secularización } \\
\text { de 4 conventos }\end{array}$ \\
\hline
\end{tabular}

Esta periodización, cuya significación o relevancia podría ser cuestionable en primera instancia, corresponde con sucesos que afectaron el proceso de desarrollo previsto por los franciscanos a su plan evangelizador, tales como el proceso de secularización que se estaba dando en todo el Nuevo Mundo y del cual Yucatán no podría escaparse, así como de los cambios de intereses políticos y económicos que la Corona tuvo durante esa época y que se reflejaron en nuevas disposiciones relacionadas con el papel que las ordenes debian asumir en la conquista espiritual. Tampoco debe dejar de tomarse en cuenta el cambio que en ellos mismos tuvieron los propios franciscanos y que transformó sus actitudes y proyectos. Y aunque al señalar los períodos del proceso de evangelización y de desarrollo de las instalaciones conventuales, no se puede ser absoluto, ni totalmente preciso, esta propuesta sí parece corresponder con las características formales de los edificios que en cada una de esas etapas se fundaron y construyeron.

Las fechas que delimitan a los períodos propuestos coinciden con algunos de los capítulos celebrados por la Orden en el convento de Mérida y en los que se formalizaba la fundación de los conventos de la región. Al mismo tiempo se ha señalado como fechas relevantes en esos períodos dos acontecimientos relacionados con la vida del fraile franciscano Diego de Landa ya que él, particularmente, se convirtió en actor importante en el proceso de erección de nuevos conventos. Su celo por proteger y conservar los derechos que la orden franciscana tenía en el territorio yucateco lo llevaron a entablar relaciones conflictivas tanto con el clero secular como con otros componentes de la sociedad de la época, particularmente con los encomenderos. El papel que desempeña cuando asume el Obispado de Yucatán es favorecedor para su Orden y el crecimiento de los territorios que ocuparon. 


\section{Tipología funcional en los diferentes períodos de fundación}

En cada uno de los períodos que hemos propuesto se fueron fundando diferentes conventos con sus respectivos pueblos de visita que asumieron también diferente función respecto al avance del proyecto general de evangelización. Tomando esto en cuenta es posible proponer una tipología funcional de los conventos en cada período:

- Tipo funcional A. Convento Central

- Tipo funcional B. Convento de enlace

- Tipo funcional C. Convento de avanzada o de penetración

A continuación describiremos en términos de su función cada uno de los cuatro períodos y los conventos que en ellos se erigieron.

\section{Período inicial (1546-1561)}

En este período se fundan 8 conventos, en Campeche (1549), Mérida (1549), Maní (1549), Conkal (1549), Izamal (1549), Sisal (1553), Calkiní (1561) y Homún (1561). (junto a cada convento aparece la fecha oficial de su fundación en el capítulo de la provincia)

Las necesidades fundamentales que estas instalaciones debían atender en esta primera etapa inicial fueron:

- Ocupación del territorio en sitios pacificados total o parcialmente, en los que se tuvieran nexos amistosos con las autoridades indigenas, tales que, que les permitieran acercarse a la población indígena con cierta seguridad y autoridad.

- Concentración de la población para un mayor control y facilidad en las tareas de conversión, bautismo y evangelización masivos.

- Y formación incipiente, pero sólida, de una red de establecimientos con posibilidades de rápida expansión.

En este período la Misión de Yucatán sería, dentro de la clasificación que propone Ricard, de ocupación y los conventos recién fundados formalmente en los capítulos celebrados en Mérida en los años de 1549, 1553 y 1561 estaban dirigidos a cumplir distintas funciones como partes de la misión, además de las funciones ineludibles de evangelización que debía de atender cualquier convento. 


\section{Tipo funcional A \\ Convento central}

El convento de Mérida era el centro administrativo, operativo y de servicios de todos los demás conventos y destinado a mantenerse con esas funciones mientras el proyecto evangelizador existiera. Era la cabecera de la región, llámese custodia, guardianía o provincia según la jerarquía obtenida en cada etapa. Funcionó como sede de la máxima autoridad entre los religiosos, punto de reunión donde se celebraban las reuniones generales, o capítulos, cada tres años y centro de formación de los frailes recién llegados. Al ser centro de servicios contó con una biblioteca y archivo, así como también con una enfermería para atender a los frailes de la región. También atendió a los pueblos de indios ubicados en las afueras de Mérida, indios traídos del centro del virreinato para la conquista de Yucatán y a los indigenas mayas ubicados en pueblos o reducciones para el servicio de los españoles de la capital.

\section{Tipo funcional B \\ Conventos de enlace}

El convento de Campeche era puerta de entrada y de enlace con las otras provincias, con la Nueva España, Cuba y España principalmente. En este convento tenían su primer contacto con el territorio yucateco quienes venían de otras tierras. Era puesto de recepción y descanso, a él llegaban los avíos necesarios para los demás conventos y las primeras noticias del exterior. En él se esperaba también el transporte que los llevaría a sus destinos, tierra adentro o hacia el exterior.

Los conventos de Izamal, Calkiní y Homún eran puntos de enlace entre los conventos de la misión. Buscaban servir de conexión segura entre el convento central en Mérida y los conventos de penetración. Recuérdese que los frailes se movían a pie y "era importante que los religiosos pudieran ir de una casa a otra sin salir de los dominios de la orden" (Ricard, La conquista espiritual $\cdots$ p.157) y que las jornadas de camino se hicieran en pocas horas y aprovechando las menos calurosas, mayormente muy de madrugada. Servían de descanso y hospedaje para los frailes viajeros y de paso para algún otro español quienes tenían prohibido pernoctar en pueblos de indios.

El convento de Izamal se encontraba sobre el Camino Real a medio trayecto hacia la villa de Valladolid y el pueblo de Sisal en un antiguo e importante centro ceremonial prehispánico.

El convento de Calkiní se encontraba sobre el Camino Real entre Mérida y Campeche en un pueblo muy grande de concentración de indios. 
El convento de Homún era el punto intermedio en el camino de Mérida hacia Maní.

\section{Tipo funcional C Conventos de avanzada}

Los conventos de Maní, Conkal y Sisal eran puestos de avanzada, instalados en sitios prehispánicos con distintos niveles de pacificación, tenían la función de afianzarse en su territorio y conseguir extenderse para ampliar las fronteras.

Maní, hacia el sureste, en territorio de los Xiu, con quienes se tenía buenas relaciones buscaba aprovecharse de estas para continuar avanzando hacia la frontera sur, hacia el territorio de la "montaña", región más inhóspita y refugio de los indígenas rebeldes.

Conkal, hacia la costa norte, cerca de Mérida, en territorios de los Cheles, pueblo amigo. Era el punto más septentrional que buscaba atender a una numerosa población indigena y con visión de servir para extender la misión rápidamente hacia el norte y noreste de la península donde se asentaba una numerosa cantidad de mayas paganos.

Sisal, hacia el este, en territorio de los Itzaes, pacificados pero beligerantes, tierra difícil pero contigua a la villa de españoles de Valladolid protegida militarmente. Era la frontera oriental, con miras a extenderse tanto hacia el norte como hacia el sur y este. La villa de Bacalar, situada muy lejos, dentro de la selva y hacia la Bahía de la Ascensión, era su próximo punto de contacto con asentamientos españoles hacia el sureste inhóspito.

\section{Período de consolidación (1562-1572)}

En este periodo se fundan 3 conventos, ubicados en Tizimín (1563), Motul (1567) y Dzidzantún (1567).

En este período los objetivos de la etapa anterior se mantienen con la diferencia de que el estado de la conquista militar ha avanzado y la posición de los españoles en general en el territorio parece ser más segura. Las estructuras de poder civil, militar y religioso se consolidan, se cuenta con obispo y gobernador. Los grandes edificios conventuales se encuentran en dinámico proceso de construcción.

La misión continúa siendo de ocupación en torno a su centro neurálgico en Mérida. Los nuevos conventos fundados en los capítulos celebrados en 1563 y 1567 tienen principalmente la función de ser puntos de penetración el territorio norte y oriente y 
transforman la función de otros más antiguos en la propia red.

\section{Tipo funcional A \\ Convento central}

El convento de la Asunción de nuestra Señora en Mérida sigue siendo el centro administrativo y de servicios de la provincia. Conservando las funciones del período anterior. El convento esta en constante construcción ampliando sus dependencias.

\section{Tipo funcional B \\ Conventos de enlace}

Los conventos de Campeche, Izamal, Calkiní y Homún continúan siendo puntos de enlace de los conventos más alejados con el convento de Mérida. Se integra a este tipo el convento de Motul que ayudaba a conectar al recién fundado convento de Dzidzantún.

El convento de Conkal que funcionaba como el punto de avanzada ubicado más al norte, comienza a funcionar como enlace de los conventos de Motul y Dzidzantún.

\section{Tipo funcional C Conventos de avanzada}

Los conventos de Maní y Sisal continúan siendo puntos de penetración hacia el sur y oriente de la península, la frontera de los territorios españoles no ha tenido hasta ahora un avance significativo hacia esas tierras dominio aún de los indigenas rebeldes.

Con la fundación de los conventos de Dzidzantún y Tizimín se dan dos pasos hacia los territorios del norte y este de la península, menos peligrosos que los otros. Pero también importantes por lo numerosa de su población indígena.

Período de crecimiento (1573-1579)

En este período se fundan 7 conventos, ubicados en Sotuta (1576), Chancenote (1576), Ichmul (1576), Tekantó (1576), Tekax (1576), Hocabá (1576) y Hecelchakán (1579).

En esta época Landa es obispo de Yucatán y aprovecha su nueva situación para apoyar el crecimiento en número de las instalaciones conventuales, incrementa en una cantidad importante el número de religiosos en funciones, recupera puestos cedidos al clero secular en el período anterior y convierte rápidamente antiguas visitas de doctrina en nuevos conventos, es la época de mayor índice de 
crecimiento ( 1.4 conventos por año, más del doble del promedio de todo el siglo XVI). La estrategia parece ser una redensificación de los establecimientos en el territorio. Sin embargo la frontera avanza apenas un poco hacia el sur y el este.

\section{Tipo funcional A \\ Convento central}

El convento de la Asunción de nuestra Señora en Mérida sigue siendo el centro administrativo y de servicios de la provincia. Conservando las funciones del período anterior. Dado el incremento de la población española y criolla y la simpatía de muchos de ellos por los franciscanos el convento atiende también los servicios religiosos que ellos requieren.

\section{Tipo funcional B Conventos de enlace}

Los conventos de Campeche, Izamal, Calkiní, Homún y Motul continúan siendo puntos de enlace y se incorpora a este grupo, el convento de Tekantó situado sobre el Camino Real a Valladolid, anteriormente funcionaba como visita del convento de Izamal. Además de servir para facilitarles a este y al convento de Sisal la comunicación con la ciudad de Mérida, descarga de tareas y permite atender a más poblaciones en la región de Izamal. También pertenecen a este tipo, el convento de Hocabá, población ubicada en el camino que conecta Izamal con el poblado de Homún, Sotuta que ayudaba a conectar al recién fundado convento de Ichmul, y Hecelchakán, situado sobre el Camino Real a Campeche entre los conventos de Calkiní y Campeche.

En todos los casos es evidente el intento de densificar la red de establecimientos del clero regular para facilitarse la comunicación y también para atender con mayor prontitud y facilidad a un mayor número de personas.

Dos conventos que hasta entonces funcionaban como puntos de avanzada, Maní y Tizimín, al fundarse otros más adentro del territorio de los mayas, al este (Chancenote) y sur (Tekax), comienzan a funcionar también como puntos de enlace hacia esos sitios más alejados.

\section{Tipo funcional C Conventos de avanzada}

Los conventos de Dzidzantún y Sisal continúan siendo puntos de penetración hacia el norte y este. El de Dzidzantún en realidad se 
encontraba en una zona ya bastante pacificada, y limitada en su expansión por la costa marina, por lo que la labor evangelizadora fundamentalmente estaba dirigida hacia la continuidad y la densificación de la red de instalaciones religiosas. Por su parte Sisal seguía siendo un punto de avanzada difícil dada su ubicación, ya que continuaba siendo frontera oriental del territorio pacificado.

En este periodo las fronteras hacia el sur parecen querer avanzar con la fundación de los conventos de Ichmul y Tekax. Anteriormente pueblos de visita de los conventos de Sisal y Maní respectivamente su conversión en conventos con monjes asignados consistía en un enorme esfuerzo por avanzar hacia los territorios rebeldes de la montaña. Por su parte la fundación del convento de Chancenote extendía hacia el noreste la frontera que antes marcaba el convento de Tizimín en territorios indígenas.

\section{Período de secularización (1580-1603)}

En este período se fundan 10 conventos, ubicados en Tixkokob (1581), Oxkutzcab (1581), Hunucmá (1581), Tinúm (1581), Tichel (1585), Umán (1585), Ticul (1591), Temax (1591), Maxcanú (1602) y Telchac (1602).

En este perío do se secularizan dos conventos, las presiones del clero secular por conseguir se les otorguen territorios bajo su control, incluyendo algunos de los ya ocupados por los regulares, generan un ambiente de tensión entre los dos sectores de la Iglesia que es trasladado a la población en general. Se suman en este conflicto los encomenderos y las autoridades representantes de la Corona quienes ven acordes a sus intereses la disminución del poder político y económico que hasta ahora han acumulado los religiosos a su favor. Aún así los franciscanos no ceden y en estos últimos veinte años del siglo XVI y principios del XVII consiguen fundar 10 conventos en los capítulos de 1581, 1585, 1591 y 1602. La mayoría de ellos son conventos que redensifican la red y conectan los conventos ya establecidos. Es en esta época cuando el procurador franciscano Fray Alonso Ponce visita la Provincia (1588).

\section{Tipo funcional A \\ Convento central}

El convento de la Asunción de nuestra Señora en Mérida sigue siendo el centro administrativo y de servicios de la provincia. Conservando las funciones del periodo anterior.

Tipo funcional B. Conventos de enlace

La mayoría de los conventos que en el período anterior funcionaban 
como de enlace continúan cumpliendo dicha función, con una sola excepción. Al secularizarse el convento de Chancenote el convento de Tizimín vuelve a quedar al extremo de la red por lo que podemos considerarlo nuevamente como convento de avanzada. Sotuta anteriormente enlace con Ichmul se seculariza y deja como más próximos contactos de Ichmul otros dos conventos de avanzada, Tekax y Sisal.

De los conventos fundados en este período 8 funcionan como enlaces, los de Tixkokob, Oxkutzcab, Tinum, Umán, Ticul, Temax, Maxcanú y Telchac. Anteriormente todos estos eran visitas de otros conventos más antiguos y se situaban en los caminos que los comunicaban entre sí.

Tixkokob y Tinum en el Camino Real a Valladolid.

Oxkutzcab en el camino a Tekax y Peto.

Umán y Maxcanú en el Camino Real a Campeche.

Ticul entre Calkiní, Maní y Oxkutzcab.

Temax y Telchac en la zona de Dzidzantún.

\section{Tipo funcional C Conventos de avanzada}

Los conventos de Sisal, Dzidzantún, Tekax e Ichmul continúan funcionando como puntos de avanzada. Tizimín vuelve a serlo ante la secularización de Chancenote en 1586.

Los otros dos se instalan en Tichel y Hunucmá.

Tichel era una población muy pequeña ubicada en la frontera sur de la península cerca de la Laguna de Términos, fue el puesto que se encontraba más alejado de los demás

Hunucmá, aunque relativamente cerca de la cabecera, se encontraba en una zona hasta entonces desatendida por los dos cleros. Su posición además era estratégica ya que se situaba sobre el importante camino a Sisal, nuevo puerto de entrada de la gubernatura además de Campeche, y cuya construcción había sido promovida por el alcalde Don Diego de Quijada desde 1563.

A continuación presentamos una tabla síntesis de la serie tipológica funcional en la que se relacionan los períodos con las funciones de las instalaciones conventuales que se fundan en cada uno de ellos y se muestran las transformaciones en el uso que tuvieron de acuerdo a la etapa a la que corresponden. 


\begin{tabular}{|c|c|c|c|c|}
\hline \multicolumn{5}{|c|}{ Serie tipológica funcional } \\
\hline \multirow[b]{2}{*}{ Localidad } & \multicolumn{4}{|c|}{ Período } \\
\hline & $\begin{array}{c}\text { Inicial } \\
1546-1561\end{array}$ & Consolidación & $\begin{array}{c}\text { Crecimiento } \\
1573-1579\end{array}$ & $\begin{array}{c}\text { Secularización } \\
1580-1602\end{array}$ \\
\hline MERIDA & Central & Central & Central & Central \\
\hline CAMPECHE & Enlace & Enlace & Enlace & Enlace \\
\hline MANI & Avanzada & Avanzada & Enlace & Enlace \\
\hline CONKAL & Avanzada & Enlace & Enlace & Enlace \\
\hline IZAMAL & Enlace & Enlace & Enlace & Enlace \\
\hline SISAL & Avanzada & Avanzada & Avanzada & Avanzada \\
\hline HOMUN & Enlace & Enlace & Enlace & Enlace \\
\hline CALKINI & Enlace & Enlace & Enlace & Enlace \\
\hline TIZIMIN & & Avanzada & Enlace & Avanzada \\
\hline DZIDZANTUN & & Avanzada & Avanzada & Avanzada \\
\hline MOTUL & & Enlace & Enlace & Enlace \\
\hline TEKANTO & & & Enlace & Enlace \\
\hline TEKAX & & & Avanzada & Avanzada \\
\hline HOCABA & & & Enlace & Enlace \\
\hline SOTUTA & & & Enlace & \\
\hline CHANCENOTE & & & Avanzada & \\
\hline $\begin{array}{c}\text { ICHMUL } \\
\text { HECELCHAKAN }\end{array}$ & & & $\begin{array}{c}\text { Avanzada } \\
\text { Enlace }\end{array}$ & $\begin{array}{l}\text { Avanzada } \\
\text { Enlace }\end{array}$ \\
\hline OXKUTZCAB & & & & Enlace \\
\hline HUNUCMA & & & & Avanzada \\
\hline TIXKOKOB & & & & Enlace \\
\hline TINUM & & & & Enlace \\
\hline TICHEL & & & & Avanzada \\
\hline UMAN & & & & Enlace \\
\hline TICUL & & & & Enlace \\
\hline TEMAX & & & & Enlace \\
\hline MAXCANU & & & & Enlace \\
\hline TELCHAC & & & & Enlace \\
\hline
\end{tabular}




\section{Las series formales}

\section{Aspectos metodológicos generales}

\section{Recursos para el estudio tipológico}

Para el análisis de los esquemas de organización formal de los partidos arquitectónicos de los conjuntos conventuales hemos recurrido a dos recursos para conocer el fenómeno estudiado. Los documentos que nos proporcionan la historiografía y la observación directa de los hechos arquitectónicos.

\section{Los documentos historiográficos}

El documento que escribió Fray Antonio de Ciudad Real en el viaje por la provincia de San José de Yucatán en 1588 acompañando al procurador franciscano Fray Alonso Ponce. En este documento se describen someramente las características físicas de las cabeceras de doctrina que existían en ese momento y que fueron visitadas por los frailes.

"El Catálogo de construcciones religiosas del Estado de Yucatán" que incluye una descripción más completa de la situación de los edificios en la primera mitad del siglo XX y los planos arquitectónicos indicando el estado en que entonces se encontraban.

Los planos arquitectónicos de que dispone el Gobierno de Campeche de los conventos que se encuentran en su territorio (Campeche, Hecelchakán y Calkiní) y la tesis de licenciatura en arquitectura de María Teresa Sansores Bernés acerca del convento de San Bernardino de Sena en Valladolid, Yucatán.

\section{La observación directa}

En visitas a los edificios y elaborando levantamientos arquitectónicos y fotográficos (1996 y 1997). Observando las características espaciales y físicoconstructivas de los conjuntos para detectar diferencias y transformaciones en los elementos constructivos y constitutivos de los espacios arquitectónicos que indiquen diferentes temporalidades y variaciones en el uso que se les ha dado.

A partir de dicha información se elaboraron los esquemas de los partidos arquitectónicos de cada uno de los conventos. Un esquema final elaborado a partir de las características físicas que encontramos en su estado reciente, y otro esquema hipotético de su situación inicial que propone las características generales básicas que presentaría a fines del siglo XVI. En aquellos casos 
de mayor complejidad en el proceso tipológico particular y en los que se contó con información suficiente fue posible elaborar un esquema hipotético intermedio en el desarrollo de su proceso evolutivo.

\section{Aspectos metodológicos particulares del análisis} de los procesos tipológicos formales

Los esquemas formales elaborados nos permiten observar similitudes y diferencias entre los partidos arquitectónicos de los distintos casos. También nos muestran las transformaciones formales ocurridas en un mismo edificio a través del tiempo y con el avance y desarrollo del proceso tipológico.

Respetando la misma categoría de análisis y los mismos elementos o variables observadas es posible estudiar el proceso tipológico de los conventos yucatecos de la segunda mitad del siglo XVI en sus dos niveles, el territorial y el temporal.

\section{Primera clasificación tipológica. Análisis territorial}

En el estudio del proceso tipológico en el territorio se analizan los objetos arquitectónicos del mismo sub-género arquitectónico que existen en un mismo tiempo o momento histórico, pero ubicados en distintos puntos del territorio.

En este nivel podrían analizarse los 28 conventos en distintas fases históricas. En este estudio analizamos la fase inicial y así se muestra en las tablas sintesis.

El estudio de los procesos de desarrollo del tipo en el territorio nos ayuda a encontrar variaciones particulares en su forma que reflejan principalmente los condicionamientos:

- Contextuales: relación con el contexto particular e inmediato, ubicación.

- Socio-funcionales: diferente función específica dentro del sistema de conventos de su región.

- Económico-políticos: diferentes condiciones o papel a desempeñar en el proceso de expansión o permanencia de la orden en el territorio.

\section{Segunda clasificación tipológica. Análisis temporal}

En este nivel se debe estudiar el proceso tipológico que muestran los edificios a través del tiempo. Se estudia el mismo objeto arquitectónico, pero en diferente momento histórico. 
Para poder estudiar este nivel es indispensable contar con información amplia del desarrollo de los objetos de estudio. Desarrollo y transformaciones ocurridas tanto en el plano constructivo como funcional. Además, también es importante conocer el panorama general de las circunstancias de la sociedad en que ocurre dicho desarrollo. Los resultados de un análisis de este nivel serían de enorme interés y utilidad en la comprensión amplia de los edificios que se estudian, pero sobrepasan las expectativas y objetivos del presente trabajo.

El estudio de este proceso de desarrollo en el tiempo nos podría mostrar las transformaciones ocurridas en un mismo edificio que reflejarían principalmente condicionamientos:

- Funcionales: transformaciones en el uso de los conventos. Diferentes actividades que desarrollaron sus usuarios.

- Socio-económicos: la importancia particular que el objeto tiene en el proceso de expansión o permanencia de la orden en el territorio. Así como su papel en las estrategias y políticas de la Orden en sus planes de permanencia en el territorio.

- Técnico- constructivo: desarrollo de las técnicas y procedimientos de construcción. Desarrollo de las habilidades constructivas.

- Repertorio: transformación en el uso de los símbolos, patrones y modelos en el diseño y construcción de la arquitectura religiosa.

\section{ANALISIS TIPOLÓGICO FORMAL DE LOS CONVENTOS YUCATECOS DEL SIGLO XVI}

Del universo total de los casos de estudio contamos con datos de algunas de las características formales que tuvieron en el siglo XVI que nos permite intentar visualizarlos o prefigurarlos y construir su esquema inicial. Pero es indispensable la información que nos proporciona su estado actual. Los planos arquitectónicos y la observación en sitio contribuyen a la concreción y definición de la imagen que los datos históricos nos proporcionan de su estado inicial.

Aunque el análisis es de carácter formal, requerimos en un principio, partir del carácter formal-funcional de los partidos arquitectónicos. Funcional porque el criterio de identificación de las zonas primarias parte de la relativa homogeneidad en la actividad general que en ella se desarrolla, y formal ya que las variables observables en los partidos arquitectónicos son relativas al carácter formal que presentan. 
En lo funcional los elementos componentes funcionales del conjunto conventual son:
a. Atrio
b. Templo
c. Capilla de indios
d. Claustro o casa
e. Huerto

En lo formal también propusimos como variables observables:

a. La presencia de algunos espacios indispensables donde desarrollar sus fundamentales tareas (componentes o zonas primarias).

b. Su figura o perfil básico

c. La disposición de dichos espacios en el conjunto unitario. Su posición respecto a los otros componentes y al todo.

d. La dimensión y proporción aproximada de los mismos.

Para hacer el estudio del proceso tipológico formal en el territorio de los 28 conventos consideramos importante emplear tres niveles:

\section{ler nivel. Los tipos formales}

- Plantear las variables observables. Estas variables son las características formales esenciales en los edificios. Nivel general.

- Elaborar el esquema inicial de cada uno de los casos.

- Observar y comparar el comportamiento de las variables.

- Proponer una primera clasificación tipológica formal a partir del análisis del punto anterior. Se obtienen así los tipos formales.

- Abstraer el "esquema esencial" (matriz) de cada uno de los tipos obtenidos de la anterior clasificación.

- Definir las características formales de los tipos obtenidos.

\section{$2^{\circ}$ nivel. Los subtipos formales}

- Para cada uno de los tipos plantear variables observables más específicas que nos permitan definir mejor la forma en cada caso. Nivel particular.

- Observar y comparar el comportamiento de estas variables en cada uno de los casos.

- Proponer una segunda clasificación tipológica formal. Se obtienen así los subtipos formales.

- Abstraer el esquema de cada uno de los subtipos obtenidos de la anterior clasificación.

- Definir las características formales de los subtipos obtenidos. 
Por carecer de información suficiente, acerca de lo construido, hemos eliminado de este análisis el caso del convento de Tichel, poblado del principio de la Colonia que estuvo ubicado diferentes sitios de la frontera suroeste, cerca de lo que hoy es la laguna de Términos.

De los 27 conventos restantes se han elaborado sus esquemas iniciales. De ellos hemos hecho una revisión del comportamiento de las variables definidas y proponemos, en una primera aproximación general, una inicial diferenciación tipológica en dos grandes tipos formales (o grupos tipológicos).

Sus esquemas podemos considerarlos los esquemas MATRIZ del convento yucateco del siglo XVI.

\section{Tipo I}

A este tipo corresponden once casos, los ubicados en las poblaciones de Mérida (1549), Campeche (1549), Maní (1549), Conkal (1549), Izamal (1549), Sisal (1553), Homún (1561), Calkiní (1561), Tizimín (1563), Dzidzantún (1567) y Motul (1567). Todos ellos fundados en los primeros 18 años del proceso de evangelización en la Península de Yucatán.

En ellos observamos que todos contaron desde sus primeros años con la presencia de los cinco componentes básicos, al menos en proyecto y en proceso de construcción.

El conjunto estaba dividido en dos grandes zonas atrio y huerto. El atrio siempre orientado al poniente y el huerto al oriente. En el atrio ubicaron la capilla abierta y en el otro territorio el templo y el claustro. El templo se ubicó al centro orientado sobre un eje oriente-poniente con el claustro en su costado norte para protegerlo del sol. Los templos tuvieron una sola nave cubierta con bóveda de cañón corrido.
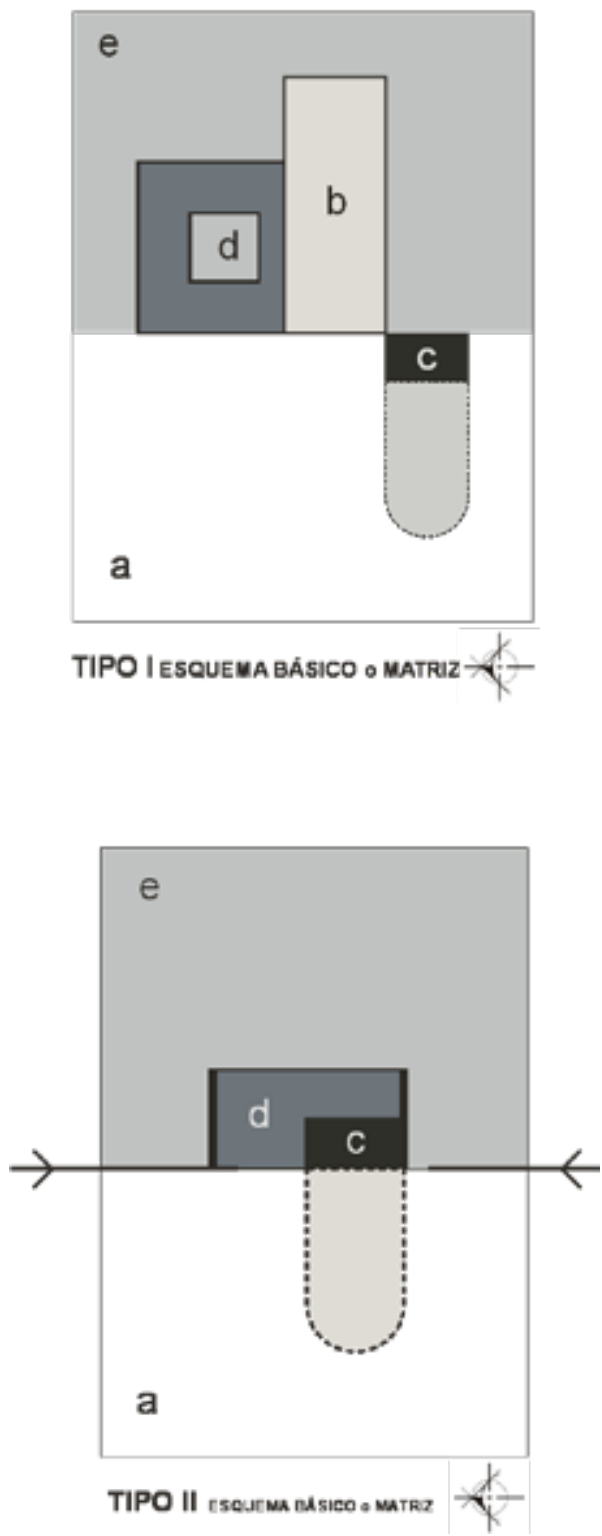
Las capillas abiertas tuvieron su presbiterio construido con mampostería adosado al claustro y la nave extendida dentro del atrio.

Algunos conventos contaron con capillas posas en las esquinas del atrio y construyeron norias sobre sus pozos para extraer agua.

Estos conventos fueron los más grandes y más consolidados de la región y permanecieron más tiempo al servicio de la Orden Franciscana.

\section{Tipo II}

A este tipo corresponden 16 casos y se encuentran en las poblaciones de Tekantó (1573), Tekax (1573), Hocabá (1573), Sotuta (1573), Chancenote (1573), Ichmul (1573), Hecelchakán (1579), Oxkutzczb (1581), Hunucmá (1581), Tixkokob (1581), Tinum (1581), Umán (1585), Ticul (1591), Temax (1591), Maxcanú (1602) y Telchac (1602).

Corresponden a las etapas de Crecimiento y Secularización. Períodos en los que los franciscanos comenzaron a tener problemas con el clero secular por el control de los territorios.

En esta época los partidos arquitectónicos de los conjuntos conventuales son más irregulares y más pequeños. Siempre conservaron su división territorial en dos grandes espacios abiertos, el atrio y el huerto. Como casi todos ellos fueron en su inicio pueblos de visita de los primeros conventos casi todos contaban con capilla abierta, con su presbiterio de mampostería y su nave de materiales perecederos en el espacio del atrio. En el territorio del huerto que fue siempre más privado se construía la casa de los frailes adosada al presbiterio, su ubicación y dimensión varió. Y a partir de esta pequeña casa posteriormente muchos de ellos construyeron sus claustros.

La orientación del conjunto siempre respetó el eje oriente-poniente para ubicar los componentes.

Los templos no se iniciaron en este período sino mucho después pero casi siempre aprovecharon los presbiterios de las capillas abiertas para sus propios presbiterios sustituyendo la construcción de madera y palma de la nave por otra de mampostería y bóvedas empleando diferentes tipos de sistemas constructivos.

A continuación presentamos dos tablas generales con todos los casos de estudio y en la que se incluye el tipo al que pertenecen, su esquema inicial y su fecha de fundación. Están ordenados cronológicamente de acuerdo a esa fecha. 


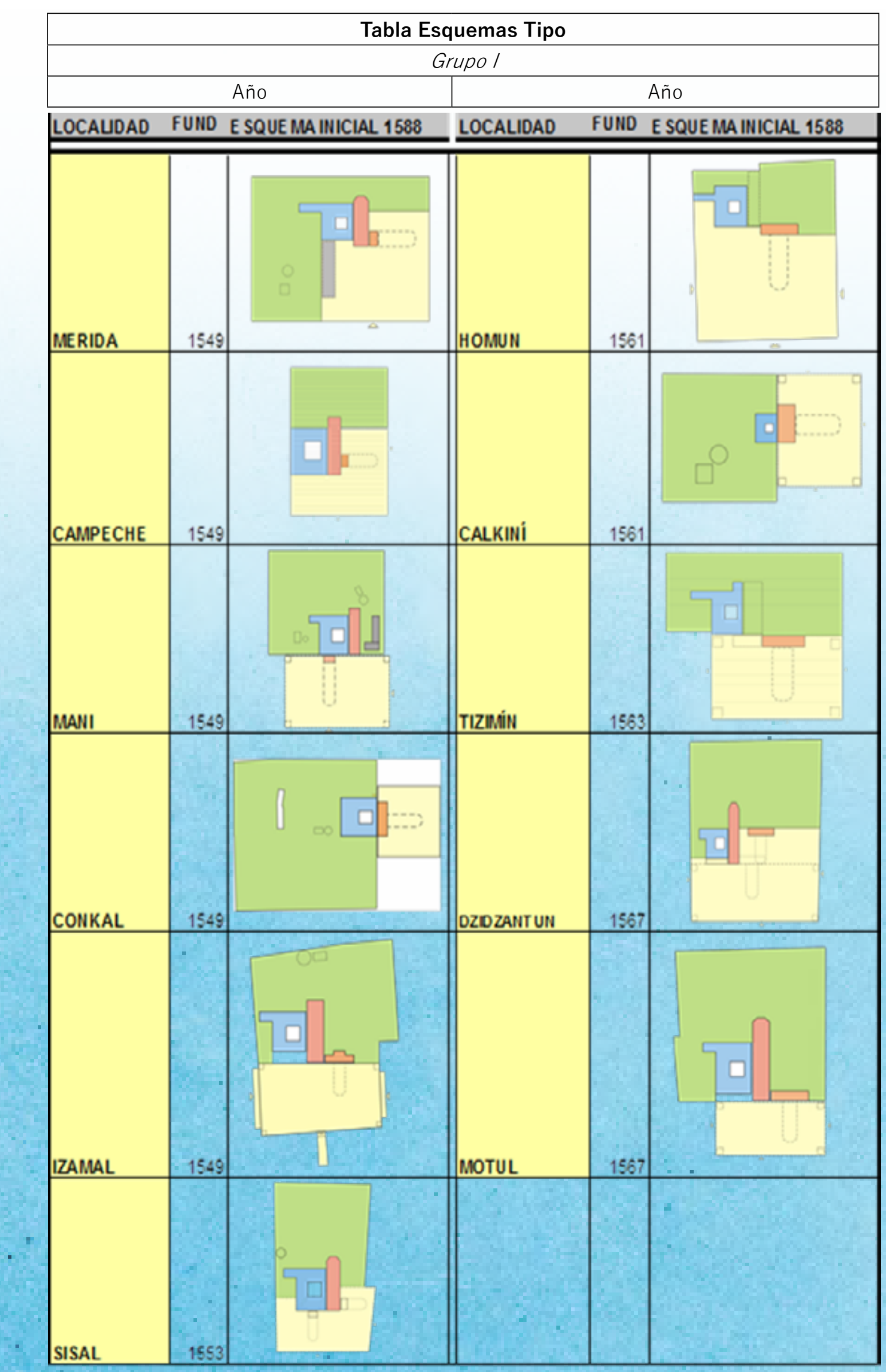




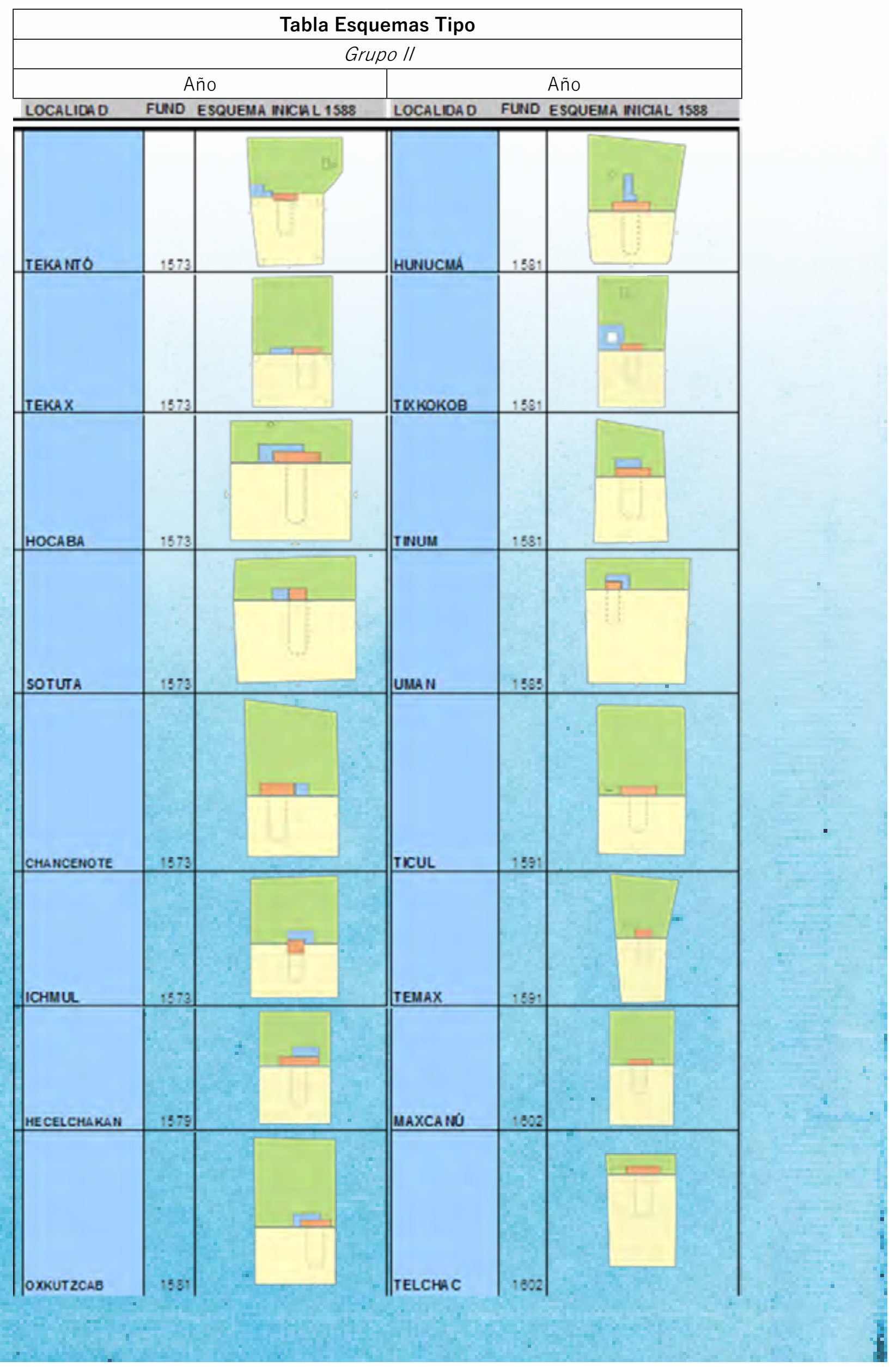




\section{CONCLUSIONES}

La investigación de los hechos histórico-arquitectónicos requiere de la participación de arquitectos que aporten la experiencia y el enfoque especializado que su formación profesional les proporciona.

La historia de la arquitectura, para convertirse en un instrumento útil y eficaz para la comprensión de los hechos arquitectónicos de nuestro patrimonio cultural regional, debe de integrarse, en los procesos de investigación, con elementos teóricos-conceptuales que le den al investigador un referente y le permitan construir un método con el cual analizar los objetos de estudio y la realidad a la que pertenecieron.

La investigación histórica arquitectónica regional requiere de sus propios enfoques, sus propias herramientas teóricas y sus propias metodologías.

El enfoque de este trabajo se ha dirigido hacia los aspectos de la tipología formal, a través del análisis del partido arquitectónico de los conventos y de las distribuciones de sus espacios en planta. Eso nos ha permitido aproximarnos a la comprensión de las relaciones entre la serie de necesidades y condiciones que forman el programa, y la configuración de los objetos arquitectónicos.

Un siguiente nivel deberá abordar con mayor amplitud lo relacionado con las funciones y las actividades desarrolladas en los espacios (serie funcional particular), para hacer corresponder los esquemas de distribución formal y espacial con los requerimientos de relación funcional interna. Estos y otros trabajos están pendientes de llevarse a cabo y dejan nuevos temas de investigación que enriquecerán el estado del conocimiento de la arquitectura religiosa regional de la Península de Yucatán. 
Revista Historia y Conservación del Patrimonio Edificado

Teoria de la Arquitectura

SOBRE EL AUTOR

Masia Astania Léón Flares

Estudio su Licenciatura y la Maestría en arquitecturan en la UADY. A cursado los Diplomados en "Teoría e Historia del Arte", "Producción de material didáctico en apoyo a la docencia", "Civilización y cultura de los mayas" y "Planeación y desarrollo para la acción tutorial" en la UADY. Obtuvo la Beca AECI en la Universidad de Zaragoza, España en 1999. Es Profesor Titular de Carrera por la UADY desde 1984. Ha sido Consejo Universitario por la UADY. Asesor, ponente y conferencista en eventos nacionales y extranjeros. A obtenido entre otros el Primer lugar en la VIII Bienal Yucateca de Arquitectura celebrada en Yucatán del año 2009, así como el Primer lugar en la X Bienal Internacional de Arquitectura celebrada en Costa Rica en el 2010 y el Reconocimiento como "Arquitecto del Año" por la UADY en Mérida del año 2012.

Año 1 No.2

29

ISSN: 


\section{REFERENCIAS}

- Arnábar Gunam, Tomás, La evangelización franciscana en Campeche, Universidad Autónoma de Campeche, Campeche, Méx., 1999, 89 p.

- Caniggia, Gianfranco, Tipologia de la edificación, estructura del espacio antrópico, Ed. Cueste, Madrid, 1995, 192 p. il.

- Gerhard, Peter, La frontera sureste de la Nueva España, Serie Espacios y tiempo 2., Trad. Stella Mastrangelo, México, UNAM, 1991, 166P. il.

- Mc Andrew, John. The open-air churches of sixteenth - century Mexico, Atrios, posas, Open chapels and other studies, Cambridge, Massachusetts, Harvard University press, 1969, 755 p.

- México-SHCP, Catálogo de construcciones religiosas del estado de Yucatán, 2 t. México, Secretaría de Hacienda y Crédito Público, Talleres Gráficos de la Nación, 1945.

- Quezada, Sergio, Pueblos y caciques yucatecos, 1550-580. El colegio de México, 1993, 227 p. il.

- Ricard, Robert, La conquista espiritual de México, Ensayo sobre el apostolado y los métodos misioneros de las órdenes mendicantes en la Nueva España de 1523-1524 a 1572, secc. Obras de Historia, México, D.F., Fondo de Cultura Económica, 1986, 491 p

- Tafuri, Manfredo, Teorías e historia de la arquitectura, Hacia una nueva concepción del espacio arquitectónico, editorial Laia, Barcelona, 1972, 287 p. il.

- Waisman, Marina, La estructura histórica del entorno, 3a. ed., Buenos Aires, Nueva Visión, 1985, (Arquitectura contemporánea) 288 p. 


\section{ANEXO I}

\begin{tabular}{|c|c|c|c|}
\hline Localidad & Año de fundacion & Periodo & Observaciones \\
\hline \multicolumn{4}{|c|}{$\begin{array}{c}1545 \\
\text { Se inicia formalmente la primera misión }\end{array}$} \\
\hline Mérida & $1549(1547)$ & \multirow{8}{*}{$\frac{A}{1546-1561}$} & \multirow[t]{2}{*}{1549 Llega Landa a Yucatán } \\
\hline Campeche & $1549(1546)$ & & \\
\hline Maní & 1549 & & \\
\hline Conkal & 1549 & & \\
\hline Izamal & 1549 & & 1552 Landa guardián de Izamal \\
\hline Sisal & 1553 & & 1556 Landa custodio \\
\hline Homún & 1561 & & 1560 Landa guardián de Mérida \\
\hline Calkiní & 1561 & & \\
\hline \multicolumn{4}{|c|}{$\begin{array}{c}1561 \\
\text { Se erige la prov. de Yuc. y Guatemala. } \\
\text { Landa, primer provincial. } \\
1562 \\
\text { Auto de fe de Mani }\end{array}$} \\
\hline Tizimín & 1563 & \multirow{3}{*}{$\frac{B}{62-1572}$} & 1562 Llega Toral a Yucatán. \\
\hline Dzidzantún & 1567 & & \\
\hline Motul & 1567 & & \\
\hline \multicolumn{4}{|c|}{$\begin{array}{c}1573 \\
\text { Landa obispo } \\
\end{array}$} \\
\hline Tekantó & 1573 & \multirow{7}{*}{$\begin{array}{c}C \\
1573-1581\end{array}$} & \\
\hline Tekax & 1573 & & \\
\hline Hocabá & 1573 & & \\
\hline Sotuta & 1573 & & \\
\hline Chancenote & 1573 & & \\
\hline Ichmul & 1573 & & \\
\hline Hecelchakán & 1579 & & 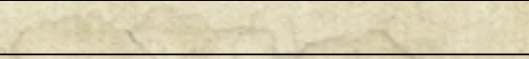 \\
\hline \multicolumn{4}{|c|}{$\begin{array}{c}1579 \\
\text { Muerte de Landa }\end{array}$} \\
\hline Oxkutzcab & 1581 & \multirow{7}{*}{$\begin{array}{c}D \\
1580-1603\end{array}$} & \\
\hline Hunucmá & 1581 & & \\
\hline Tixkokob & 1581 & & \\
\hline Tinum & 1581 & & \\
\hline Tichel & 1585 & & No existe actualmente \\
\hline Umán & 1585 & & \\
\hline Ticul & 1591 & & \\
\hline Temax & 1591 & & \\
\hline Maxcanú & 1603 & & \\
\hline Telchac & 1603 & & \\
\hline & Secularización d & Apo de co & \\
\hline
\end{tabular}




\section{ANEXO II}
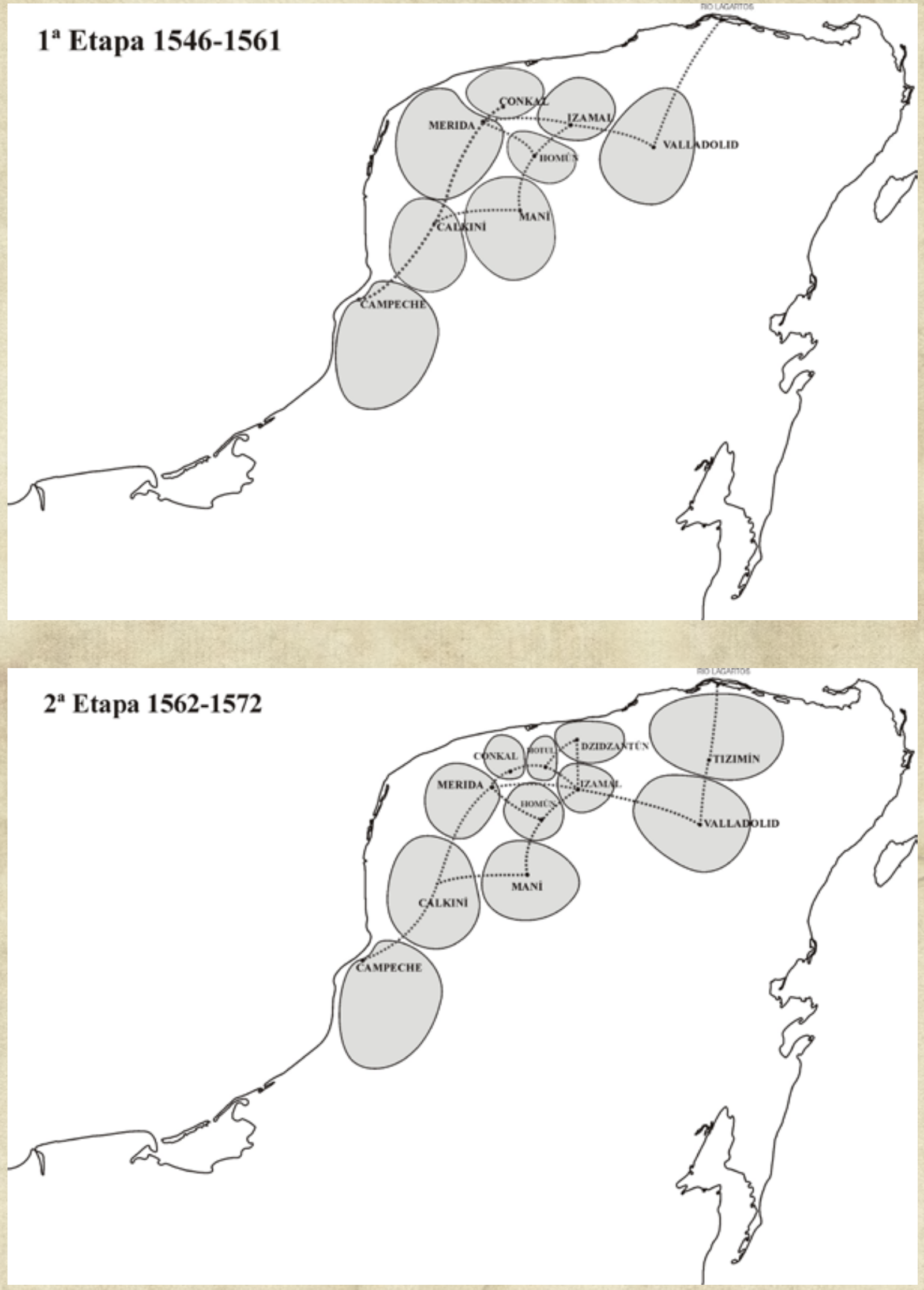

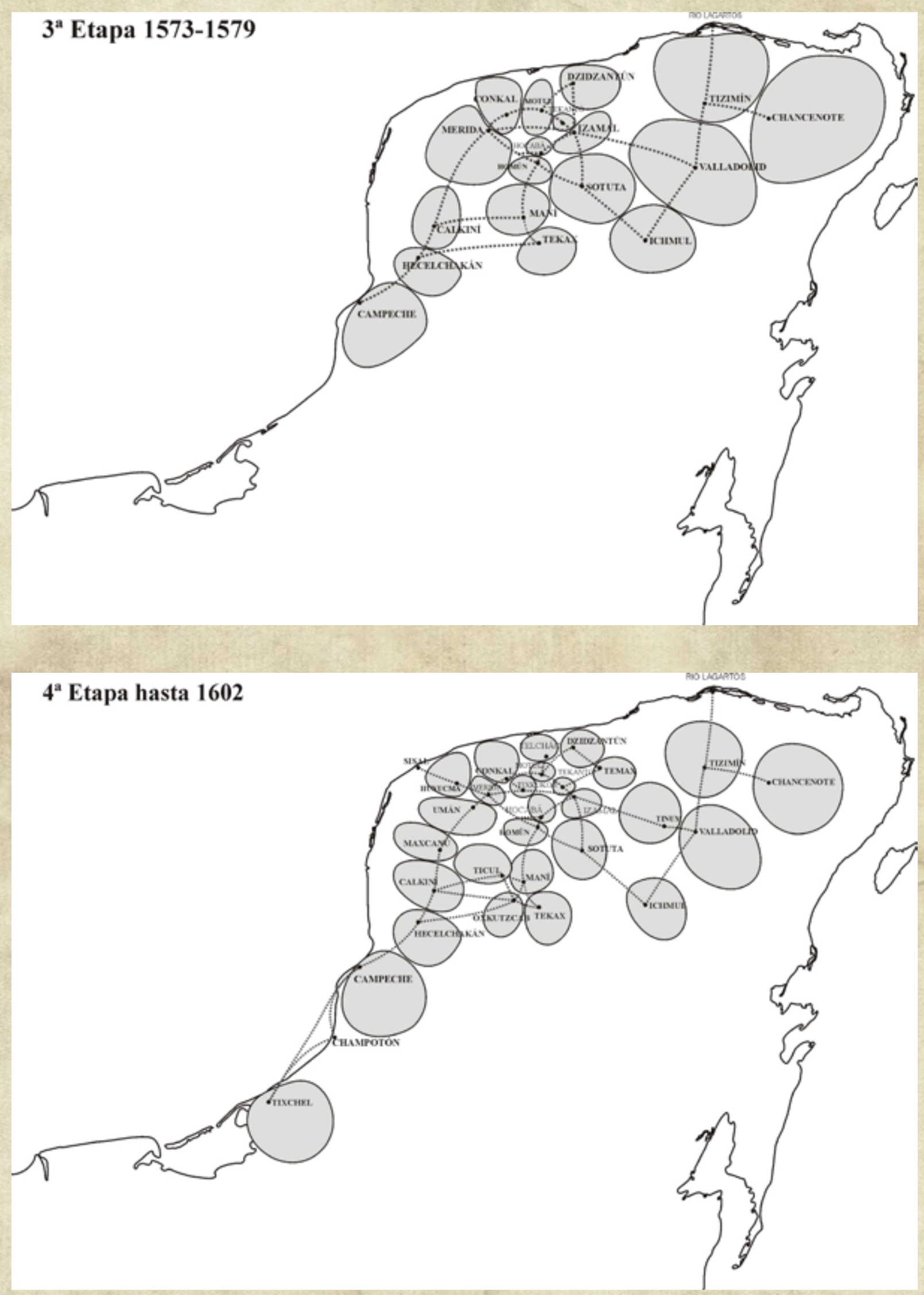